\title{
Konservative Orthopädie in der Sportmedizin
}

\section{Epidemiologie, Diagnose, aktuelle Präventions- und Therapiekonzepte}

\author{
F. Mayer, J. Weber, M. Cassel \\ Hochschulambulanz der Universität Potsdam, Zentrum für Freizeit-, Gesundheits- und Leistungssport
}

Sowohl dem Breiten- und Gesundheitssport als auch dem Einsatz körperlicher Aktivität in der Prävention und Therapie unterschiedlicher Krankheitsbilder des Stützund Bewegungssystems kommt aus ärztlicher Sicht eine steigende Bedeutung zu. Zudem ist mittlerweile uneingeschränkt nachgewiesen, dass körperliche Aktivität maßgeblich am Erhalt und der Wiederherstellung von Lebensqualität in allen Alters- und Leistungsklassen beteiligt ist. Dennoch ist die Umsetzung dieser Kenntnisse in der
Bundesrepublik - trotz wachsender Akzeptanz - nicht abschließend und flächendeckend gelungen und sollte weiter kommuniziert werden.

Ziel der folgenden Übersicht ist die Vermittlung ausgewählter Aspekte aktueller sportorthopädischer Daten zur Epidemiologie von Beschwerden und Verletzungen im Sport, einer sinnvollen und begründeten Diagnostik sowie derzeitiger Therapie- und Präventionskonzepte.

\section{Einleitung}

Das öffentliche Interesse an unterschiedlichen Sportarten ist ungebrochen. Im Fokus der Medien stehen dabei insbesondere Großveranstaltungen wie Welt- und Europameisterschaften in den traditionellen Sportarten Fußball, Leichtathletik, Tennis, aber auch Rudern, Kanu und Golf. Häufig wird dem Spitzensport eine erhöhte Verletzungs- und Überlastungsinzidenz oder -prävalenz zugeschrieben, wenngleich diese Annahme im Ausmaß oft überschätzt wird und aus wissenschaftlicher Sicht nicht abschließend belegt ist. Unter Berücksichtigung der Trainingsbelastung und des Trainingsumfangs lässt sich dagegen nicht selten eine geringere Beschwerderate bei erwachsenen und Nachwuchsleistungssportlern nachweisen. Im Spitzensport existiert ein bundesweites (sport)medizinisches Versorgungssystem, organisiert durch Bund und Länder, welches die Gesunderhaltung von Athleten aller Altersklassen zum Ziel hat.

Die zunehmende Anzahl an körperlich Aktiven in Breiten- und Gesundheitssport kann durch aktuelle Zahlen gut belegt werden. So ist beispielsweise bekannt, dass die Anzahl an Läufern in den vergangenen Jahren stark zugenommen hat. Im Nachwuchsbereich ist insgesamt eine Zunahme weiblicher Athletinnen zu verzeichnen (Abb. 1). Auch die Anzahl derjenigen Personen, die regelmäßig Krafttraining betreiben, hat zugenommen. Bezogen auf die Gesamtbevölkerung sind jedoch bislang nur rund $1 / 4$ und, bezogen auf ältere Menschen über 60 Jahre, nur rund 10 - 15\% im Sinne einer regelmäßigen

\section{Abkürzungen}

ACL anterior cruciate ligament (vorderes Kreuzband)

CT Computertomografie

DOSB Deutscher Olympischer Sportbund

EMG Elektromyografie

MRT Magnetresonanztomografie

MTT Medizinische Trainingstherapie

Kräftigung aktiv. Aus sportorthopädischer Sicht ist somit auch im Breiten- und Gesundheitssport eine evidenzbasierte Versorgung mit dem Ziel der raschen Wiederherstellung bzw. Aufrechterhaltung der sportlichen Belastbarkeit sicherzustellen.

In verschiedenen Bereichen der Prävention und Rehabilitation von Erkrankungen des Stütz- und Bewegungssystems ist Sport bzw. körperliche Aktivität als eine zentrale Intervention anerkannt und wissenschaftlich validiert. So findet sich beispielsweise eine Reduktion von Stürzen und Verletzungen nach einem sensomotorischen Training, eine geringere Abnahme der Knochendichte nach axialer Belastung knöcherner Strukturen durch Sprünge oder eine Reduktion der Schmerzsymptomatik bei Fibromyalgiepatienten durch Krafttraining bzw. bei chronisch-unspezifischen Rückenschmerzen durch Bewegung. 


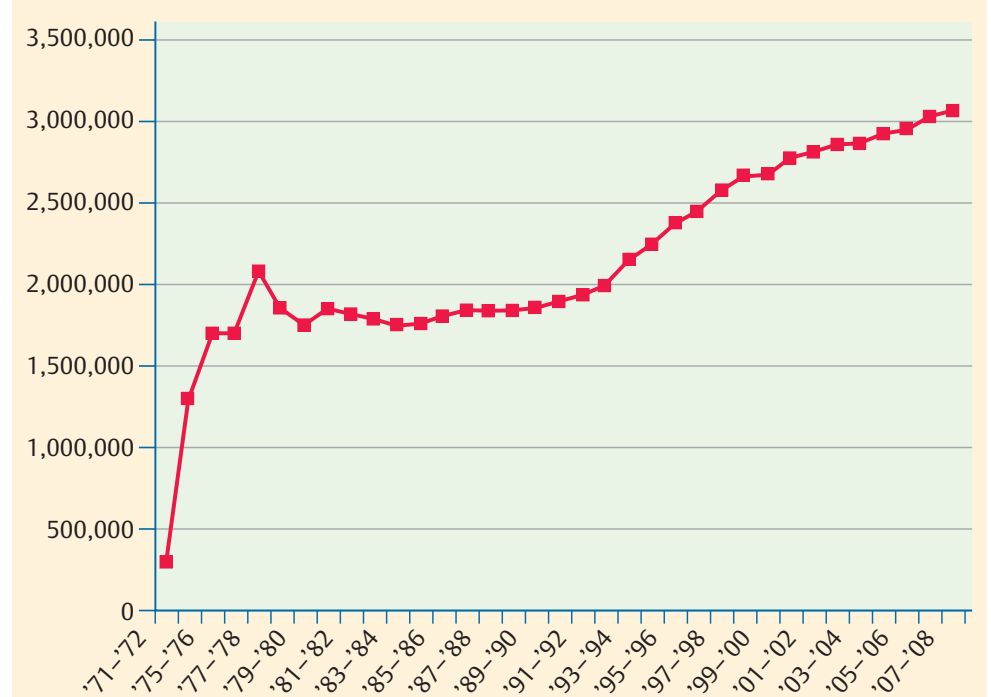

Source: National Federation of State High School Associations.

Participation numbers were not available for the 1972-1973 or 1974-1975 school years. Knowles S B Br J Sports Med 2010; 44; 38-44

Abb. 1 - Zunahme der weiblichen Athleten im Nachwuchssport.

\section{Häufige Verletzungen und Beschwerden im Breiten- und Spitzensport}

Das Risiko für Sportverletzungen wird in epidemiologischen Arbeiten unterschiedlich bewertet. Differenziert werden muss insbesondere zwischen

- Art,

- Häufigkeit und

- Intensität der sportlichen Aktivität.

Aussagekräftige (wissenschaftliche) Daten zu Häufigkeiten beziehen sich in der Regel auf die Anzahl der Sporttreibenden pro Sportart und die Zeit der tatsächlichen Sportausübung. Als Inzidenz wird dabei die Anzahl neu aufgetretener Beschwerden (üblicherweise pro Jahr) bezeichnet. Unter der Prävalenz wird die Anzahl an Verletzungen zu einem Zeitpunkt oder in einem Zeitraum verstanden. Insgesamt treten rund 2 Sportverletzungen pro 100 Sporttreibende pro Jahr auf.

Als Sportverletzung wird dabei eine akute Überlastungssituation mit Überschreiten der physiologischen Belastungsgrenzen einzelner Gewebe definiert.

Definition

Sportverletzung

akute Überlastungssituation mit Überschreiten der physiologischen Belastungsgrenzen einzelner Gewebe
Die Verletzungsrate ist bei Männern etwa doppelt so hoch wie bei Frauen. Am häufigsten treten Sportverletzungen im Alter zwischen 5 und 25 Jahren auf. Epidemiologische Arbeiten zeigen ein hohes relatives Risiko für Ball- und Mannschaftssportarten (z. B. Volleyball, Handball, Fußball, Basketball). In rund 70\% der Fälle ist die untere Extremität betroffen.

- Als häufigste Sportverletzung (rund $25 \%$ aller Fälle) gilt das Supinationstrauma des oberen Sprunggelenks.

Bei Kindern sind Frakturen relativ häufig, bei Erwachsenen zwischen 20 - 50 Jahren Distorsionen (z.T. mit Bandverletzungen) und Muskelverletzungen, bei Älteren gewinnen Frakturen erneut an Bedeutung. Knieverletzungen treten im Frauensport vorrangig und in Spielsportarten wie Fußball, Basketball häufiger auf (z.T. 40\% höhere Verletzungsrate) (Renström et al. 2008). Auch der Schweregrad von Knieverletzungen wird bei Athletinnen meist höher eingestuft. Neben den Knieverletzungen nehmen Schädel-Hirn-Verletzungen an Bedeutung zu. Man geht derzeit von rund 11 Kontaktverletzungen/100000 sportlichen Einsätzen in Spielsportarten aus.

Von Sportverletzungen abzugrenzen sind Überlastunsgbeschwerden und Sportschäden. Bezüglich Sportschäden wird mit einer Häufigkeit von 1:4000 Sportler ausgegangen. Für die breite Masse der Pathologien ist die Effizienz verschiedener Präventions- und Rehabilitationsprogramme nicht abschließend geklärt.

- Männer verletzen sich im Sport etwa doppelt so häufig wie Frauen. Dagegen sind schwere Knieverletzungen (v.a. ACL-Verletzungen im Nachwuchssport) bei weiblichen Sportlerinnen häufiger als bei männlichen Athleten.

Verletzungen und Beschwerden des Stütz- und Bewegungssystems sind in der Regel verantwortlich für einen Trainings- und Wettkampfausfall und die weitere sportliche Belastbarkeit. Besonders bedeutsam ist dies im Kindes- und Jugendalter. Allerdings ist die Datenlage bezüglich der Erfassung von Beschwerden im Kindesund Jugendalter derzeit noch lückenhaft. Verschiedene Statistiken weisen auf eine hohe Variabilität der Verletzungsrate pro Jahr hin (zwischen 10 und 70 Verletzungen pro 1000 Stunden Exposition, je nach Sportart), wobei auch hier Spielsportarten im Vordergrund stehen. Die Extremitätengelenke (v.a. Schulter, Ellenbogen, Knie) sind dabei vorrangig bei Belastungen mit hoher Bewegungsgeschwindigkeit gefährdet. Eine chirurgische Intervention ist im Vergleich zu Erwachsenen eher selten. Zu berücksichtigen sind insbesondere Apophysenund Epiphysenverletzungen. Daten aus dem Nachwuchsbereich zeigen zudem, dass bei Athletinnen (bei insgesamt geringerer Inzidenz) ein höherer Schwere- 
grad von Knieverletzungen (insbesondere ACL-Rupturen) nachweisbar ist. Je nach Sportart ist bei 14-18-jährigen Athletinnen ein bis zu 8-fach erhöhtes Risiko für eine vordere Kreuzbandruptur bekannt. Insbesondere „non-contact"-Situationen mit valgischer Beinachse und Innenrotationsstellung des Oberschenkels bei Landeund Richtungswechseln werden dafür verantwortlich gemacht (Renström et al. 2008).

Seit einigen Jahren stehen durch Sport verursachte Gehirnerschütterungen bei Kindern und Jugendlichen im Mittelpunkt des Interesses. Bekannt ist ein in der Regel höherer Schweregrad bei längerer Regenerationszeit verglichen mit Erwachsenen. Epidemiologische Daten zeigen eine Prävalenz von rund $10 \%$ aller Verletzungen im Nachwuchssport. Als Ursache wird insbesondere eine nicht ausreichend kräftige Nackenmuskulatur diskutiert.

- Sportbedingte Gehirnerschütterungen sind im Kindesund Jugendalter häufig und bedingen eine längere Regenerationszeit, verglichen mit erwachsenen Athleten.

Die Analyse von Sportverletzungen und Sportschäden bei internationalen Großveranstaltungen wie Weltmeisterschaften oder Olympischen Spielen ist von öffentlichem Interesse. Hierbei wird häufig angenommen, dass im Spitzensport eine höhere Verletzungsrate vorliegt, wenngleich gesicherte und publizierte wissenschaftliche Daten erst in jüngerer Zeit verfügbar sind (Alonso et al. 2009; Junge et al. 2009). Verletzungsmechanismus, Beschwerdeart und -lokalisation sind von der Sportart bzw. der jeweiligen Disziplin abhängig. So ergab die Verletzungs- und Beschwerdeanalyse der IAAF-Weltmeisterschaften 2007 in Osaka 192 Verletzungen und Überlastungsbeschwerden bei 1660 Athleten aus 49 Nationen (97 Verletzungen auf 1000 akkreditierte Athleten). $80 \%$ der Fälle betrafen die untere Extremität, wobei Überlastungsbeschwerden im Vordergrund standen. Besonders häufig waren Athleten aus Zehnkampf, 10000-m-Lauf der Frauen, 3000-m-Hindernislauf der Frauen, Fünfkampf und Marathon der Männer betroffen (Alonso et al. 2009).

Die Analyse der medizinischen Daten der Olympischen Spiele in Peking ergab insgesamt 1055 Verletzungen entsprechend einer Verletzungsrate von 96,1 pro 1000 Athleten (häufigste Diagnosen: Supinationstrauma $n=81$, Oberschenkelverletzungen $n=75$ ). Zweidrittel der Verletzungen traten während Wettkämpfen auf. Athleten und Athletinnen aus Spielsportarten waren am häufigsten betroffen. Rund 55\% der Diagnosen betrafen die untere Extremität, rund 20\% die obere, 15\% den Rumpf, $12 \%$ Kopf und Hals. Lediglich 41 Verletzungen führten zu einem Trainings- oder Wettkampfausfall von mehr als 4 Wochen (Junge et al. 2009).

Bezüglich der Ursachen und Risikofaktoren von Sportverletzungen und Sportschäden wird von einer
Tabelle 1

Mögliche Einflussfaktoren für Sportverletzungen und Überlastungsschäden.

\begin{tabular}{|c|c|c|}
\hline Einflussfaktor & Differenzierung & Bewertung \\
\hline Achsabweichungen des Skeletts* & & +o \\
\hline Athletenalter* & & oо \\
\hline \multirow[t]{2}{*}{ Belastungsdosierung** } & Trainingsumfang & ++ \\
\hline & Intensität & $t_{0}$ \\
\hline Material und Trainingsumgebung** & & oо \\
\hline muskuläre Flexibilität (Dehnfähigkeit)* & & oо \\
\hline (neuro)muskuläre Insuffizienz* & & +0 \\
\hline Schuhwerk** & & oo \\
\hline Sportart** & $\begin{array}{l}\text { höhere Verletzungsrate in } \\
\text { Spiel- und Kraftsportarten }\end{array}$ & +o \\
\hline Trainingsalter* & & ++ \\
\hline Untergrund ${ }^{* *}$ & & oo \\
\hline Vorverletzung* & & ++ \\
\hline $\begin{array}{l}\text { * intrinsisch; }{ }^{* *} \text { extrinsisch; ++: durch wi } \\
+ \text { +o: hohe Praxisrelevanz, wissenschaftlich } \\
\text { geklärt; oo: Praxisrelevanz }\end{array}$ & $\begin{array}{l}\text { senschaftliche Untersuchun } \\
\text { nicht abschließend und für a }\end{array}$ & $\begin{array}{l}\text { elegt } \\
\text { portarten }\end{array}$ \\
\hline
\end{tabular}

multifaktoriellen Genese ausgegangen und zwischen intrinsischen (personenbezogenen) und extrinsischen Einflüssen differenziert (Tab. 1). Wissenschaftlich belegt sind allerdings nur wenige Erklärungskonzepte, wie beispielsweise eine steigende Inzidenz von Laufsportbeschwerden bei einem wöchentlichen Trainingsumfang von mehr als $60 \mathrm{~km}$. Belegt ist darüber hinaus, dass das Vorbestehen einer Verletzung oder einer Überlastungsreaktion das relative Risiko für deren Wiederauftritt erhöht. Schließlich ist bekannt, dass bei geringem Trainingsalter Sportverletzungen und Überlastungsschäden häufiger sind.

Neben dem Geschlecht wird zunehmend die Bedeutung des Körpergewichts als eine der Ursachen für ein hohes Verletzungsrisiko diskutiert. Strittig ist hierbei insbesondere, ob das erhöhte Gewicht und damit die erhöhte Last an sich für ein gering gesteigertes Risiko verantwortlich ist, oder ob nicht eher eine damit meist vergesellschaftete unzureichende posturale Kontrolle bei reduzierten sensomotorischen Fähigkeiten als Grund anzunehmen ist. 
Tabelle 2

Beispiel einer systematischen Sport- und Trainingsanamnese für aktuelle Beschwerden und eine Kaderuntersuchung.

\begin{tabular}{|c|c|}
\hline Anamnese-Item & $\begin{array}{l}\text { Beispiel (chronisch-unspezifische Rücken- } \\
\text { beschwerden, männlich, Kanurennsport) }\end{array}$ \\
\hline Personen-ID/Untersuchungs-ID & $0001 / 0005$ \\
\hline Untersuchungsdatum & 01.08 .2011 \\
\hline Name, Vorname & Mustermann, Max \\
\hline Geburtsdatum & 01.01.1990 \\
\hline Geschlecht & männlich \\
\hline Kaderstatus/Leistungsklasse & B-Kader/Nationalmannschaft \\
\hline $\begin{array}{l}\text { aktuelle Anamnese } \\
\text { (Beschwerden, Vorerkrankungen, } \\
\text { zurückliegende Therapien, } \\
\text { Medikamente, Voroperationen, } \\
\text { Familienanamnese) }\end{array}$ & $\begin{array}{l}\text { rezidivierende Beschwerden im unteren Rücken, } \\
\text { v. a. nach Belastung und am nächsten Tag } \\
\text { keine Ausstrahlung } \\
\text { keine sensomotorischen Defizite } \\
\text { erstmaliges Auftreten } 03 / 11 \text {, seither } 2 \text { Episoden, } \\
\text { jeweils } 3 \text { Tage } \\
\text { keine Therapie } \\
\text { keine Medikamente } \\
\text { keine Voroperationen }\end{array}$ \\
\hline $\begin{array}{l}\text { Trainingsanamnese } \\
\text { (Trainingsalter, Sportarten, } \\
\text { Disziplinen, Umfang, } \\
\text { Intensität) }\end{array}$ & $\begin{array}{l}\text { Kanurennsport (Kajak) seit } 11 \text { Jahren } \\
\text { derzeit rund } 15 \text { Trainingseinheiten pro Woche } \\
\text { (dabei } 6 \text { TE Krafttraining, } 4 \text { TE Ausdauertraining } \\
\text { an Land) } \\
\text { derzeit Wettkampfsaison, hohe Intensitäten }\end{array}$ \\
\hline
\end{tabular}

Besondere Bedeutung ist dem Auftreten von Rückenschmerzen im Sport beizumessen. In der überwiegenden Mehrzahl der Fälle gehen diese mit einer eingeschränkten körperlichen Belastbarkeit einher und sind zudem einer der häufigsten Gründe für krankheitsbedingte Arbeitsausfälle. Die Prävalenz über die gesamte Lebenszeit in der Allgemeinbevölkerung wird mit bis zu $90 \%$ angegeben (Bono 2004). Die Punktprävalenz allein für den unteren Rückenschmerz (Low Back Pain) beträgt 13,4-28,7\% für unterschiedliche Länder, die Rezidivrate nach Erstinzidenz 35 - 80\% (Choi et al. 2010). Daten aus dem Spitzensport zeigen eine Prävalenz von rund $75 \%$ und eine jährliche Erstinzidenz von rund 5\%. Die Ätiologie unterliegt strukturellen, funktionellen und psychologischen beziehungsweise psychosozialen Faktoren, welche sich gegenseitig verstärken und miteinander in Wechselwirkung stehen können. Derzeit ungeklärt ist, ob eine Kombination an Defiziten auf neuronaler, muskulärer und/oder struktureller Ebene zu einem erhöhten Risiko führt. So ist bekannt, dass nicht jede strukturelle Anomalie mit Beschwerden einhergeht, sofern sie muskulär-funktionell kompensiert wird. Selbst für nach- weisbare strukturelle Pathologien (z. B. segmentale Instabilitäten) ist der Einfluss auf Beschwerden und die sportliche Leistungsfähigkeit, auf funktionelle (z. B. neuromuskuläre Stabilisationsfähigkeit, Kraftausdauer) sowie psychologische bzw. psychosoziale Einflussfaktoren (z.B. Schmerzempfindung), nicht determiniert.

- Die Prävalenz von Rückenbeschwerden ist bei Athleten geringer als in der Allgemeinbevölkerung.

\section{Die sportorthopädische Untersuchung und Diagnostik}

Die sportorthopädische Untersuchung unterscheidet sich im Wesentlichen nicht von einer üblichen orthopädischen Diagnostik des Stütz- und Bewegungsapparats. Unterschieden werden muss aber grundsätzlich zwischen einer Untersuchung, die im Rahmen einer Vorstellung aufgrund von Beschwerden erfolgt und einer sog. Gesundheits- oder Tauglichkeitsuntersuchung im Breiten- und Spitzensport. Stellt sich ein Athlet mit Beschwerden in der Sprechstunde vor, erfolgt die Untersuchung meist lokal, wohingegen bei einer Tauglichkeitsuntersuchung das gesamte muskuloskelettale System erfasst wird.

Das deutsche Betreuungssystem im Spitzensport umfasst auf nationaler Ebene Athleten mit Bundeskaderstatus und auf Länderebene den Nachwuchsbereich in den jeweiligen Schule-Leistungssport-Verbundsystemen. Die jährliche Untersuchung der Bundeskaderathleten erfolgt an sog. lizensierten medizinischen Untersuchungszentren des Deutschen Olympischen Sportbundes (DOSB), die sportmedizinische Betreuung im Beschwerdefall meist über die Spitzenverbände (Verbandsärzte und Kooperationsärzte) oder die Olympiastützpunkte und deren kooperierende Ärzte. Auf Länderebene ist die Versorgung unterschiedlich organisiert, wird aber in der Mehrzahl der Fälle in Absprache mit den Ministerien und den Landessportverbänden ebenfalls von ausgewiesenen sportmedizinischen Zentren an Universitäten, sportmedizinischen Schwerpunkten an Kliniken bzw. niedergelassenen Orthopäden, Unfallchirurgen und Allgemeinmedizinern mit der Zusatzbezeichnung Sportmedizin sichergestellt. Unabhängig von der Art der Untersuchungen bedeutsam sind einerseits die gründliche Erfassung einer sportartspezifischen Anamnese zur Erfassung der körperlichen Belastung und andererseits eine eindeutige Beurteilung der Belastbarkeit und der Reintegration in den Sport (return-to-play). Schließlich sind evidenzbasierte, validierte Präventionsund ggf. Therapiekonzepte einzuleiten. 


\section{Anamnese}

Die Anamnese von Athleten wird analog einer Patientenanamnese erfasst und richtet sich entweder nach den angegebenen Beschwerden oder im Falle einer Gesundheitsuntersuchung nach der Erfassung möglicher Erkrankungen, die die sportliche Belastbarkeit einschränken könnten. Unfallmechanismus und Art der Belastung weisen bereits in der Mehrzahl der Fälle auf die Diagnose hin oder lassen die Differenzialdiagnose deutlich einschränken. Darüber hinaus können beschwerdeprädisponierende Merkmale abgeleitet werden.

Im Rahmen der Sport- und Trainingsanamnese werden Sportart, Disziplin, Trainingsumfang, -häufigkeit und -alter erfasst. Die aktuelle Belastung bzw. die bei aktuellen Beschwerden vorliegende Belastungsart, -intensität sowie der Umfang werden erfragt (Tab. 2). Es folgt die Dokumentation von Beschwerden bei Belastung und deren bisherige Behandlung. Im Rahmen einer Gesundheits- oder Kaderuntersuchung werden zusätzlich die vegetative Anamnese, die subjektive Leistungsfähigkeit, die Ermüdbarkeit und Regenerationsfähigkeit erfasst. Berufliche, schulische oder familiäre Belastungssituationen sind von Bedeutung. Des Weiteren werden das Gewicht, Gewichtsveränderungen (insbesondere in Sportarten mit Gewichtsklassen) sowie die Verwendung von Nahrungsergänzungsmitteln und/oder Medikamenten erfragt.

\section{Klinische Untersuchung}

Bei Beschwerden erfolgt die körperliche Untersuchung analog der orthopädischen Patientenuntersuchung in den Abschnitten Inspektion, Palpation und Funktionsprüfung (Abb. 2, Abb. 3). Je nach Indikation werden darüber hinaus bildgebende Verfahren eingeleitet. Eine Besonderheit der sportorthopädischen, klinischen Diagnostik ist dabei sicherlich die Zuordnung von Untersuchungsbefunden zu sportartspezifischen Krankheitsbildern (z. B. Druckschmerz an der Tuberositas Gerdii bei Tractus-iliotibialis-Syndrom, Druckschmerz am mediokaudalen Kalkaneus bei Plantarfasziitis).

Die sportorthopädische Tauglichkeits- oder Gesundheitsuntersuchung umfasst in der Regel zunächst die Bestimmung von Körpergröße und Körpergewicht. Bei Kindern und jugendlichen Sportlern kann zusätzlich die Erfassung des körperlichen Entwicklungsstadiums sinnvoll sein. Es folgt die Inspektion im aufrechten Stand von allen Seiten (u. a. Beurteilung der Achsen, Seitendifferenzen und Ausprägung der Muskulatur). Bei der Inspektion der Wirbelsäule lassen sich Seitabweichungen in der Frontal- und/oder Sagittalebene und eine mögliche Skoliose (z. B. Lendenwulst, Rippenbuckel bei Inklination) erkennen. Allgemeine Funktionstests (z. B.

\section{An Herrn}

Max Mustermann

Am Musterring 1

12345 Musterhausen

Sehr geehrter Herr Mustermann,

Wir berichten über Ihre sportmedizinisch-orthopädische Untersuchung vom 01.08.2010.

Vorgeschichte: Sportmedizinische Grunduntersuchung, Sportart: Leichtathletik/Wurf, B-Kader. Derzeit keine Beschwerden im Bereich des Stütz- und Bewegungsapparats. Keine Voroperationen, Z.n. Fraktur li. Kleinzehe 99. Vor 3 Jahren rezidivierend HWS-Beschwerden, nach konservativer Behandlung beschwerdefrei. Keine Medikamente, keine Nahrungsergänzungsmittel.

Befund: $\quad$ 20-jähriger Athlet, freies und flüssiges Gangbild. Hockstellung, Zehenund Fersenstand uneingeschränkt möglich. WS gerade, kein Druckund Klopfschmerz, unauffällige obere Extremitäten. Genua Vara um 2 QF. Ansonsten unauffällige untere Extremitäten. Keine sensomotorischen Defizite.

Röntgen: HWS 2 Ebenen: Harmonische Lordose, keine Einengung der Intervertebralräume, keine Abstützreaktionen, keine pathologischen Auffälligkeiten

Diagnosen: $\quad$ 1.Z.n. rez. HWS-Syndrom

2. Genua vara

3. Z.n. Fraktur li. Kleinzehe 99

Procedere: Aus sportmedizinisch-orthopädischer Sicht ergeben sich keine Einschränkungen für die ausgeübte Sportart. Auf eine fortwährende Kräftigung der HWS- und Schulterblattmuskulatur wird hingewiesen. Sinnvoll ist hierbei zunächst eine physiotherapeutische bzw. trainingstherapeutische Anleitung, anschließend Übernahme in das sportartspezifische Training.

Mit freundlichen Grüßen

Dr. Max Mäxchen

(Ärztl. Direktor)

Abb. 2 - Beispiel einer Dokumentation (Arztbrief einer sportorthopädischen Ganzkörperuntersuchung, versendet an Athleten und ggf. betreuende Ärzte).

Hockstellung, Zehengang und -stand, Fersengang und -stand, Finger-Boden-Abstand) schließen sich an. Nur begrenzt abschätzbar sind die segmentale und komplexe Stabilität sowie die Funktionsfähigkeit einzelner Wirbelsäulenabschnitte unter Belastung. Die Palpation der Muskulatur dient der Erfassung von Tonusveränderungen. Die neurologische Diagnostik differenziert radikuläre von pseudoradikulären Symptomen.

Die Beurteilung der Extremitätengelenke findet durch die Erfassung der aktiven und passiven Beweglichkeit (Neutral-Null-Methode), die Palpation (z.B. Sehnenansätze) und Funktionstests (z. B. ImpingementTests der Schulter, Prüfung der Bandstabilität oder Meniskuszeichen am Knie) statt. Bisher nicht abschließend geklärt ist, welche (klinischen) diagnostischen Variablen zielsicher und evidenzbasiert in Präventionsmaßnahmen abgeleitet werden können. Dies betrifft sowohl den Spitzensport als auch die Allgemeinbevölkerung. 


\section{Grundlagen}

SPORTÄRZTLICHER UNTERSUCHUNGSBOGEN

KLEINE ORTHOPÄDIE (BEWEGUNGSAPPARAT)

$\begin{array}{lll}\text { Name, Vorname } & \text { Geburtsdatum } \\ \text { Hauptsportart, Kader } & \text { Untersuchungsdatum }\end{array}$

\begin{tabular}{|c|c|c|c|}
\hline \multicolumn{2}{|c|}{ Befund: ohne } & \multirow{2}{*}{$\begin{array}{l}\text { mit } \\
\square\end{array}$} & \multirow{2}{*}{$\begin{array}{l}\text { Bemerkung/Befund } \\
\text { höher } \quad \text { links } \square \quad \text { rechts } \square\end{array}$} \\
\hline Beckenstellung & $\square$ & & \\
\hline $\begin{array}{l}\text { Wirbelsäule (Haltung, } \\
\text { Krümmungsverhalltnisse) }\end{array}$ & $\square$ & $\square$ & \\
\hline Wirbelsäulenbeweglichkeit & $\square$ & $\square$ & mit Befund $\square$ Hws $\square$ Bws $\square$ sws \\
\hline Hüftgelenk & $\square$ & $\square$ & \\
\hline Kniegelenk & $\square$ & $\square$ & \\
\hline Sprunggelenk/Füße & $\square$ & $\square$ & \\
\hline Untere Gliedmaßen (sonstiges) & $\square$ & $\square$ & \\
\hline Schulter & $\square$ & $\square$ & \\
\hline Ellenbogen/Unterarm & $\square$ & $\square$ & \\
\hline Hand/Finger & $\square$ & $\square$ & \\
\hline Obere Gliedmaßen (sonstiges) & $\square$ & $\square$ & \\
\hline Hypermobilität/Bänderschwäche & $\square$ & $\square$ & \\
\hline Muskelstatus & $\square$ & $\square$ & \\
\hline Beschwerden & & & \\
\hline
\end{tabular}

Beschwerden

Operationen

Präventive oder therapeutische Maßnahmen

Sporttauglichkeit

Vorstellung beim Orthopäden des zuständigen OSP

Ja $\square \quad$ Nein $\square$

Untersuchender Arzt

Unterschrift des Arztes

Abb. 3 - Orthopädischer Untersuchungsbogen des Deutschen Olympischen Sportbundes (DOSB). 


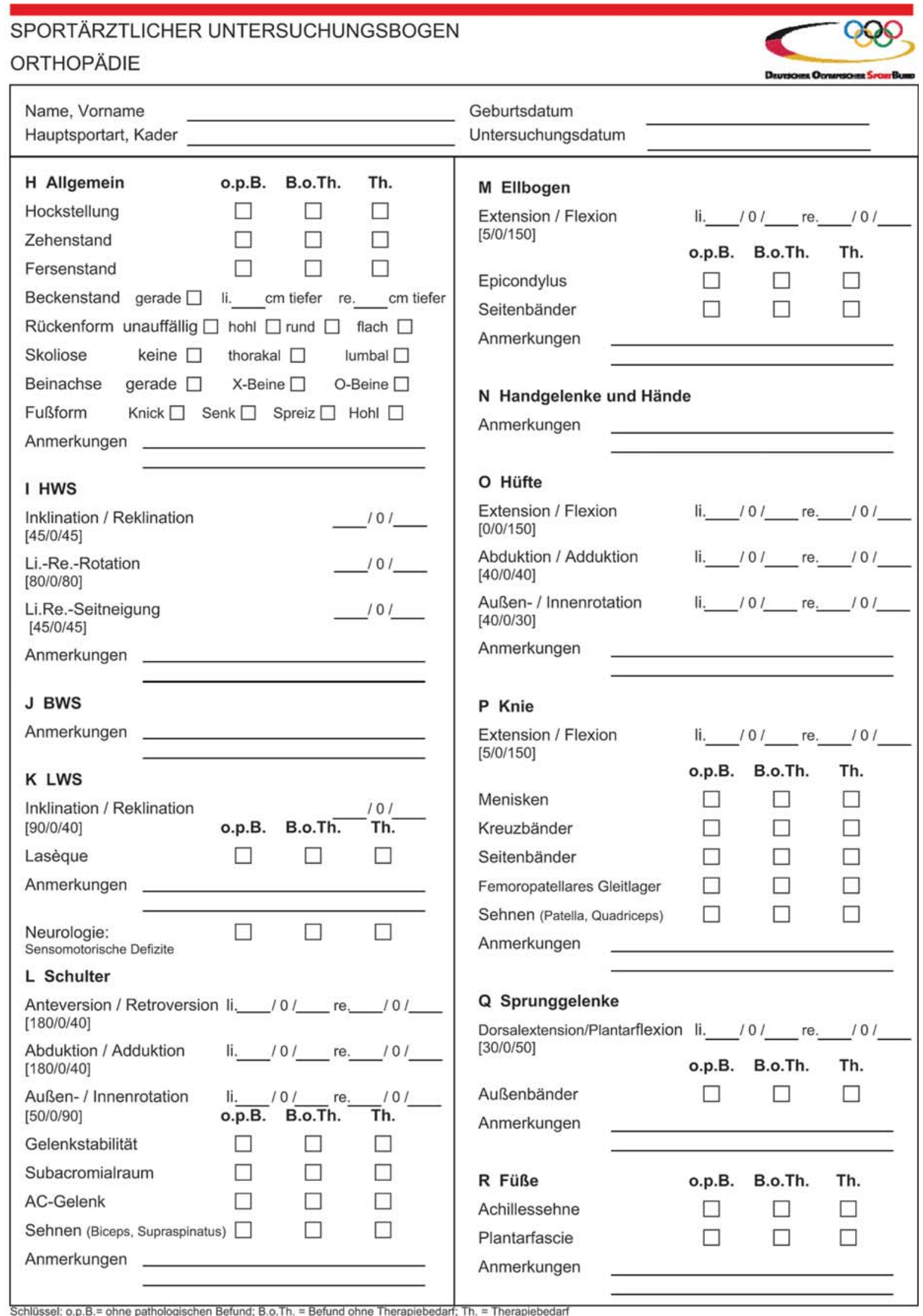




\section{SPORTÄRZTLICHER UNTERSUCHUNGSBOGEN}

ORTHOPÄDIE

Name, Vorname

Hauptsportart, Kader

\section{R ORTHOPÄDISCHE RÖNTGENAUFNAHMEN}

Schlüssel: Eb. $=$ Ebene $(\mathrm{n})$

$\mathrm{wEb} .=$ weitere Ebenen

$5010=$ Finger od. Zehen $2 \mathrm{~Eb}$.

$5011=$ Finger od. Zehen wEb.

$5020=$ Handgelenk $2 \mathrm{~Eb}$.

$5020=$ Mittelhand $2 \mathrm{~Eb}$.

$5020=$ alle Finger einer Hand $2 \mathrm{~Eb}$.

$5020=$ Sprunggelnk $2 \mathrm{~Eb}$.

$5020=$ Fußwurzel $u$. Od. Mittelfuß 2

$5020=$ Kniescheibe $2 \mathrm{~Eb}$.

$5021=5020 \mathrm{wEb}$.

$5030=$ Oberarm $2 \mathrm{~Eb}$.

$5030=$ Unterarm $2 \mathrm{~Eb}$.
$5030=$ Ellenbogengelenk $2 \mathrm{~Eb}$.

$5030=$ Oberschenkel $2 \mathrm{~Eb}$.

$5030=$ Unterschenkel $2 \mathrm{~Eb}$.

$5030=$ Kniegelenk $2 \mathrm{~Eb}$.

$5030=$ Hand od. Fuß 2 Eb.

$5030=$ Gelenke Schulter $2 \mathrm{~Eb}$

$5030=$ Beckenteilaufnahme $2 \mathrm{~Eb}$.

$5030=$ Kreuzbein od. Hüftgelenk 2 Eb.

$5031=5030 \mathrm{wEb}$.

$5040=$ Beckenübersicht
$5100=$ HWS 2 Eb.

$5101=$ HWS $w E b$.

$5105=$ BWS od. LWS je Teil 2 Eb.

$5106=$ BWS od. LWS je Teil wEb.

$5110=$ Ganzaufnahme Wirbelsäule od. einer Extremität

$5111=$ Ganzaufnahme Wirbelsäule od. einer Extremität wEb.

$5120=$ Rippen einer Thoraxhälfte $1 \mathrm{~Eb}$ $5120=$ Schulterblatt od. Brustbein $1 \mathrm{~Eb}$. $5121=5120 \mathrm{wEb}$.

\section{Anlagen}

s. Arztbrief

Hinweise für Athlet, Trainer und Verbandsarzt / auffällige Befunde, präventive oder therapeutische Maßnahmen, Prognose, Sporttauglichkeit: 
- Durch die Kombination aus einer ausführlichen medizinischen Anamnese, einer Trainingsanamnese und der körperlichen Untersuchung lassen sich rund $90 \%$ der Diagnosen im Sport feststellen.

\section{Bildgebende Verfahren}

Der Einsatz bildgebender Verfahren richtet sich nach dem klinischen Befund, der körperlichen Untersuchung und dient der Klärung der Differenzialdiagnose. Die konventionelle Röntgenuntersuchung, die Sonografie und bei spezieller Indikation die Computertomografie (CT) bzw. die Magnetresonanztomografie (MRT) werden hierzu angewandt (Abb. 4, Abb. 5). Für die (meist im Vordergrund stehende) Beurteilung von Knochen- und Gelenkstrukturen ist die Anfertigung konventioneller Röntgenaufnahmen in 2 Ebenen sinnvoll (i.d. R. bei Angabe von Beschwerden oder klinischen Auffälligkeiten). Die dynamische Untersuchung von Gelenken, Muskeln und Sehnen (auch unter Last) gelingt mit der Sonografie (hochauflösende Schallköpfe bis $15 \mathrm{MHz}$ ).

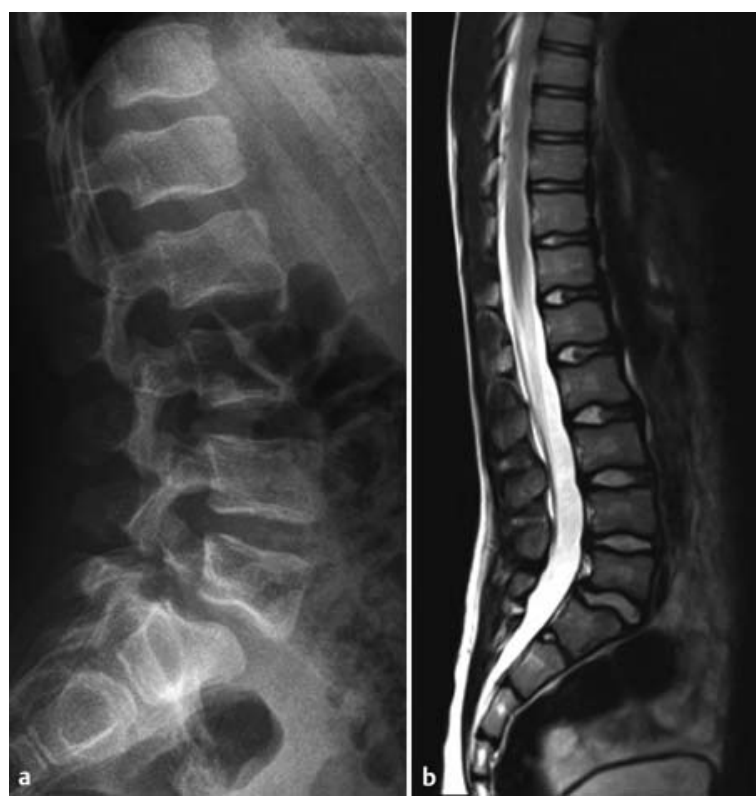

Abb. 5 - Darstellung einer im Rahmen der Jahresgrunduntersuchung erfassten Spondylolisthese L5/S1 eines Nachwuchsathleten. a Nativröntgen, LWS seitlich im Stehen. b Korrespondierendes MRT im Liegen.
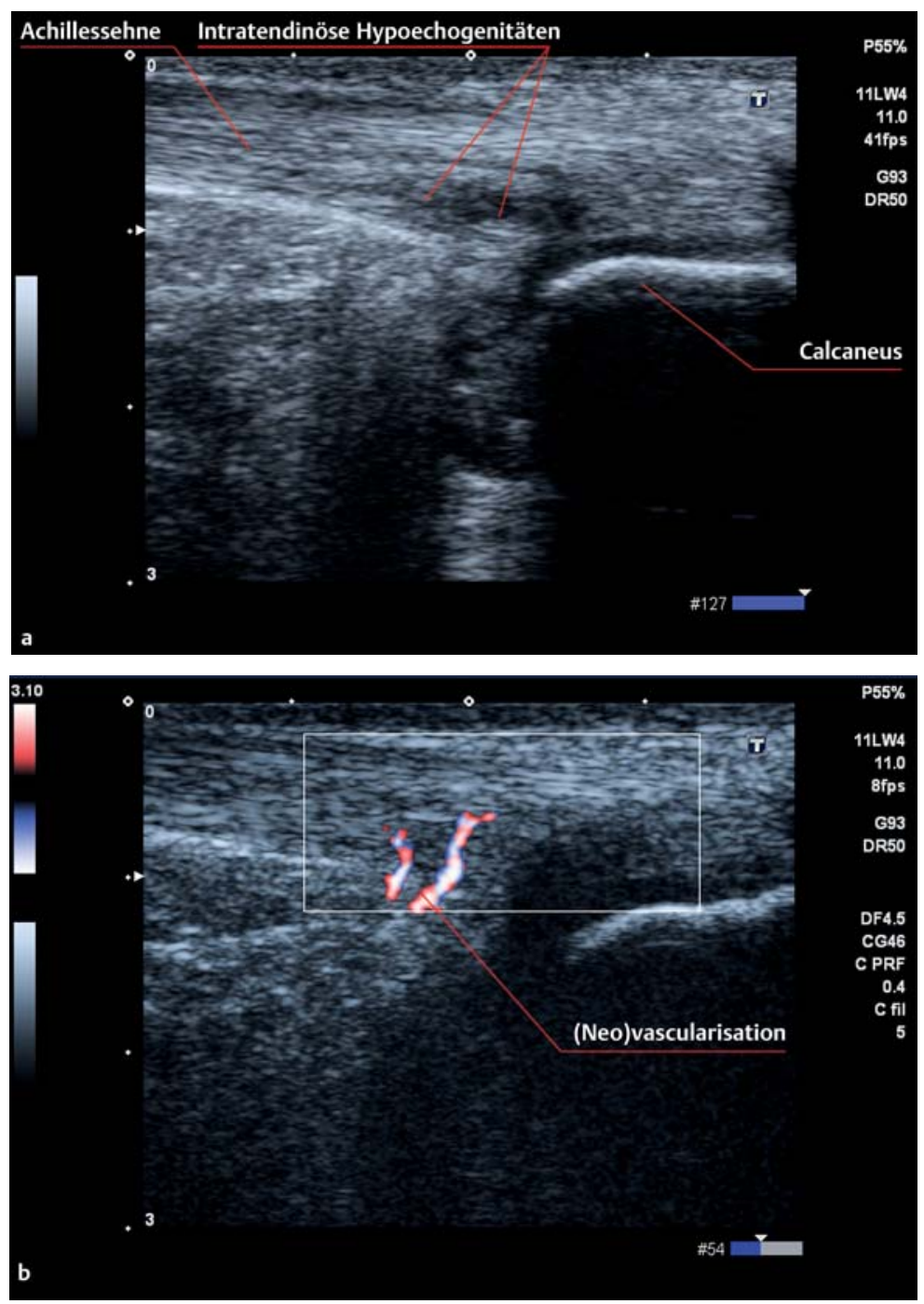

Abb. 4 - Sonografie (a) und Powerdopplersonografie (Advanced Dynamic Flow Modus, b). Darstellung einer Tendinopathie der Achillessehne einer erwachsenen Läuferin.

Additive biomechanische Diagnoseverfahren können im Einzelfall und bei spezieller Indikation sinnvoll sein (Abb. 6). Ziel ist die Ergänzung der klinischen Untersuchung bei Breiten- und Spitzensportlern sowie bei Kindern und Älteren durch valide und reliabel erfasste Daten (z. B. Kraftdefizite) zur Ableitung von Präventionsstrategien (z. B. Beurteilung der Standstabilität). Eingesetzt werden Verfahren zur Kraftdiagnostik (u.a. funktionelle Krafttests mit Hanteln, isokinetische Testverfahren), kinetische (z. B. Messungen der Bodenreaktionskraft und der plantaren Druckverteilung) und kinematische Verfahren (Goniometrie, Videoanalysen, 3-D-Bewegungsanalysen) sowie Methoden zur Erfassung der Koordination und Bewegungsregulation (z.B. EMG, Posturografie). Meist bleibt die ausführliche orthopädisch-biomechanische Diagnostik speziell dafür ausgerichteten Einrichtungen vorbehalten. 

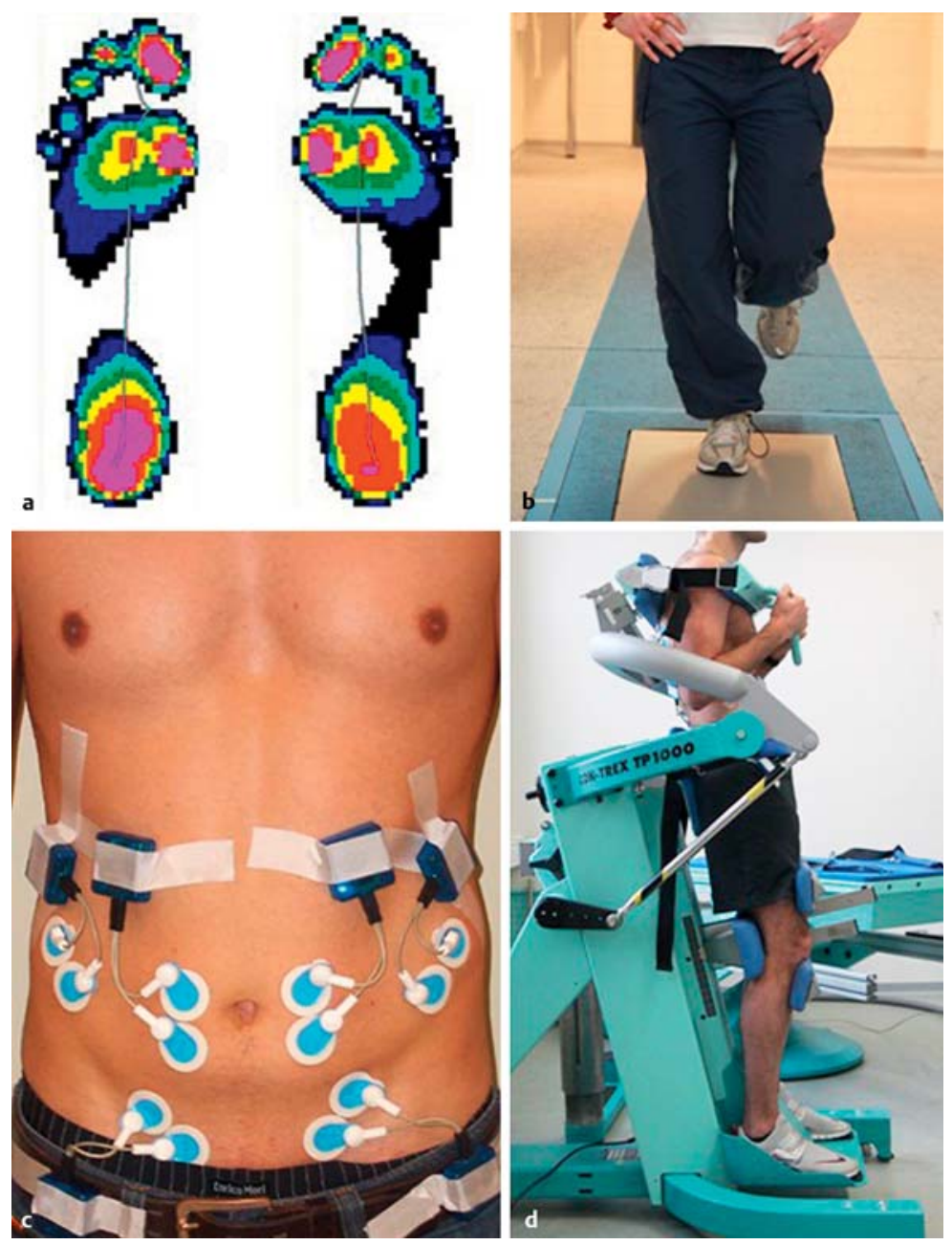

Abb. 6 - Biomechanische Mess- und Analysemethoden in der Sportorthopädie. a Ergebnis einer plantaren Druckverteilungsmessung. b Messung der Bodenreaktionskraft im Einbeinstand. c Telemetrische Messung der muskulären Aktivität (Oberflächen-EMG) am Rumpf. d Isokinetische Rumpfkraftmessung (Flexion/Extension).

\section{Grundzüge ausgewählter Therapie- konzepte in der (konservativen) Sportorthopädie}

\section{Frühfunktionelle Behandlung}

Als Erstmaßnahme nach Traumata im Bereich des Stützund Bewegungsapparats findet auch in der modernen Medizin die PECH-Regel (Pause, Eis, Compression, Hochlagern) weiterhin Anwendung. Die Ausbildung ausgedehnter Schwellungszustände bzw. Hämatome in der Umgebung der verletzten Strukturen sollte nach Möglichkeit minimiert werden. Elastische Verbände unter moderatem Zug oder Bandagen sowie ergänzend Tape-Verbände bzw. Orthesen sind sinnvoll einzusetzen. Nach zeitnaher ärztlicher Vorstellung mit Anamnese, klinischer Untersuchung und zielgerichteter bildgebender Diagnostik (Sonografie, Röntgen, MRT, CT) erfolgt die zügige Einleitung eines Therapiekonzepts. Sofern frühzeitig eine Fraktur ausgeschlossen werden kann, ist die schmerzadaptierte Vollbelastung (meist) möglich und in der Mehrzahl der Fälle sinnvoll.

Die Indikation zur operativen Behandlung erfolgt im Einzelfall nach Abwägung konservativer Behandlungsmethoden, die im Rahmen der Sportorthopädie nicht selten überlegen sind. Bei Überlastungsbeschwerden (z.B. Tendinopathien, Shin splints, muskuläre Beschwerden, knöcherne Stressreaktionen) wird in der Mehrzahl der Fälle eine Belastungsreduktion unter die Schmerzgrenze empfohlen. Eine orale und/oder lokale (Infiltrationen, Salben) antiphlogistische bzw. analgetische Therapie ist oft indiziert. Die Domäne der weiteren Vorgehensweise obliegt in der Regel physio- und trainingstherapeutischen Maßnahmen (s.u.). Durch Verwendung schützender und stabilisierender Hilfsmittel (Cast- oder Gipsverbände, Orthesen oder auch TapeVerbände) unter Schonung der verletzten Struktur ist bereits frühzeitig eine aktive Rehabilitation (Medizinische Trainingstherapie) möglich, wobei neurophysiologische Techniken im Vordergrund stehen.

- Eine längere Phase der Immobilisation sollte vermieden werden (Kontrakturen, nach wenigen Tagen einsetzende Muskelatrophie, Verschlechterung der intra- und intermuskulären Koordination).

In der frühfunktionellen physio- und trainingstherapeutischen Behandlung gewannen vor allem neurophysiologische Trainingsformen (heute meist als sensomotorisches Training bezeichnet, s. Hintergrundbox) an Bedeutung. Über eine progrediente Erhöhung des Schwierigkeitsgrads der labilen bzw. instabilen Situation und/oder Steigerung der Last können gezielt Reize mit dem Ziel der Verbesserung von intra- und intermuskulärer Koordination gesetzt werden. Eine erhöhte funktionelle Gelenkstabilität und ein signifikant reduziertes Risiko von erneuten Verletzungen konnte dabei nachgewiesen werden.

Für Spitzensportler sind frühfunktionelle Therapiekonzepte von hoher Relevanz, da jede unnötige Verzögerung zu einem Trainings- bzw. Wettkampfausfall mit deutlichen Einschränkungen der sportartspezifischen Leistungsfähigkeit führt. Der stetig wachsende Druck von Seiten des Umfelds, der Medien und der Sponsoren erwartet darüber hinaus eine (nicht immer sinnvolle) zeitliche Optimierung der medizinischen Behandlung. Schließlich sind eine enge Zusammenarbeit zwischen Arzt und Therapeut sowie klare Vorgaben durch den behandelnden Sportorthopäden, insbesondere in der Frühphase der Behandlung, von essenzieller Bedeutung. 
Abb. 7 - Sensomotorisches Training zur Erhöhung der funktionellen Gelenkstabilität des OSG bzw. der unteren Extremität (geordnet nach Schwierigkeitsgraden von oben links nach unten rechts)

Unter einem sensomotorischen Training wird die gezielte Modulation sensorischer (afferenter) Information mit folgender in der Regel willkürlicher, motorischer (efferenter) Umsetzung unter Last verstanden. Am weitesten verbreitet ist diese Form des Trainings bei Übungen auf unterschiedlichen Untergründen zur funktionellen Stabilisation des oberen Sprunggelenkes (Abb. 7). Mittlerweile ist das sensomotorische Training auch in der Rehabilitation und Prävention von Kniegelenkverletzungen (z. B. in der Nachbehandlung von ACL-Rupturen), Schulterver- letzungen (z. B. rezidivierende Subluxationen) und bei Wirbelsäulenbeschwerden (z. B. chronisch-unspezifische untere Rückenschmerzen) anerkannt. Belegt werden konnte unter anderem eine Zunahme der Kraft, eine Erhöhung der muskulären Aktivität bei Belastung und nach Perturbation. Als Hilfsmittel werden verschiedene Formen von labilen und instabilen Unterlagen (u. a. Minitrampolin, Pads, Kreisel, Weichmatten, Bälle) eingesetzt. Eine Übertragung der Trainings- und Übungsposition in Situationen des täglichen Lebens (situationsangepasstes Training wie z. B. Richtungswechsel unter Last, plötzliche Körperstabilisation) ist erstrebenswert. Somit empfiehlt es sich bereits unter standardisierten Bedingungen im Rahmen der Prävention und Rehabilitation unter funktioneller Belastung (z. B. an der Treppe, Sprungund Stabilisationsbelastungen ggf. unter additiver Gewichtsbelastung) zu trainieren. Eine Trainingshäufigkeit mit mindestens 3-5 Trainingseinheiten pro Woche mit mindestens 15 Wiederholungen pro Übung wird derzeit empfohlen.
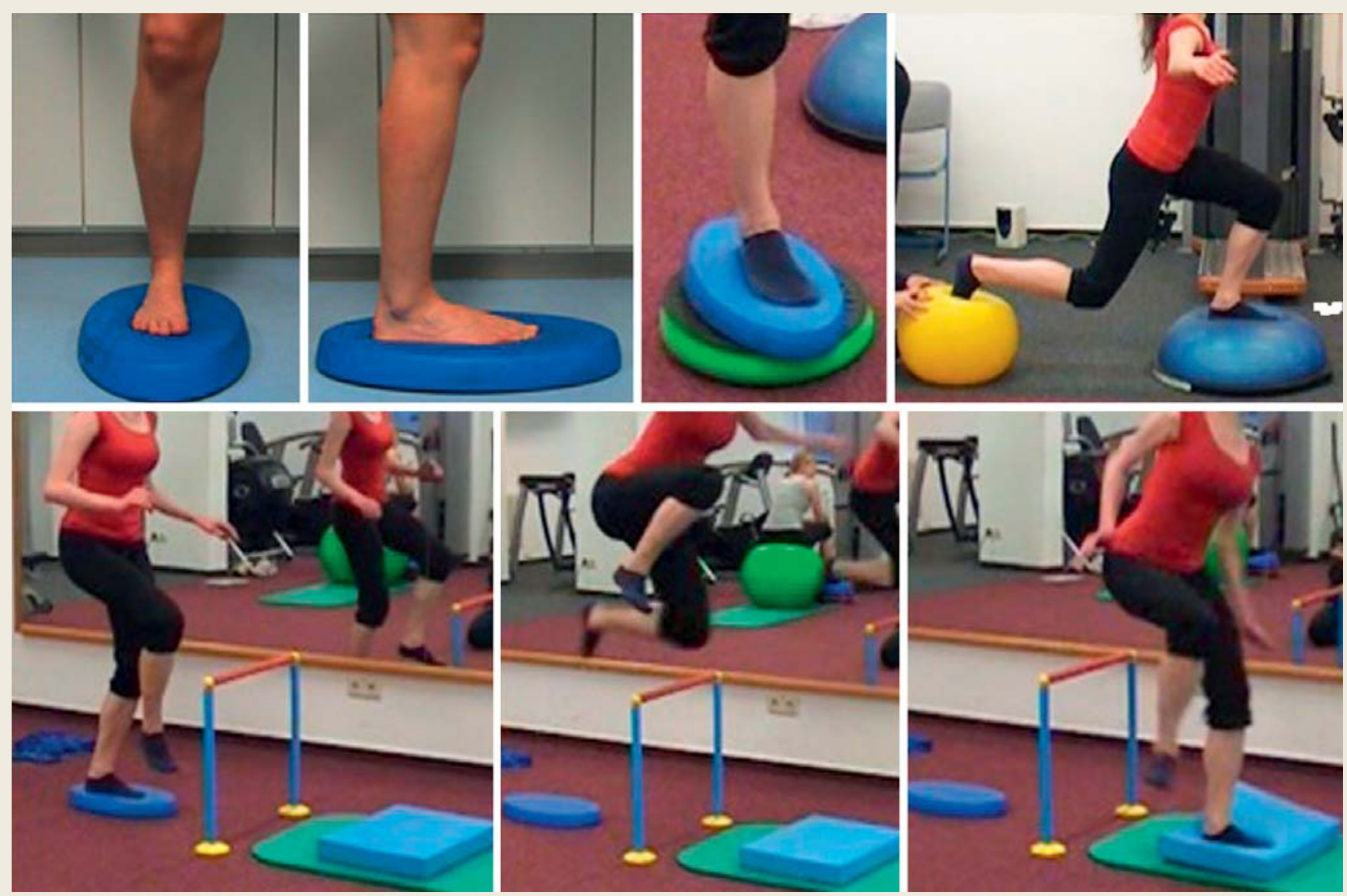

\section{Physiotherapie, Medizinische Trainingstherapie, Sporttherapie}

Physiotherapeutische und sporttherapeutische Interventionen kommen in der Nachbehandlung akuter Verletzungen und in der Therapie von Überlastungsschäden des Stütz- und Bewegungsapparats zum Einsatz. Vorrangiges Ziel ist die Wiederherstellung der Belastbarkeit und funktioneller Bewegungsabläufe. Im Mittelpunkt steht die möglichst zügige Reintegration des Athleten in das sportartspezifische Training. Fundierte Kenntnisse bezüglich sportartspezifischer Belastungs- und Bewegungsmuster sind somit für Therapeut und Sportorthopäden unabdingbar.

$\mathrm{Zu}$ Beginn der Therapie stehen - in Abhängigkeit des Krankheitsbilds - zumeist physiotherapeutische Behandlungsformen zur Beschwerdereduktion und Abschwellung (Kryotherapie, Lymphdrainage, Ultraschall, 
Abb. 8 - Sensomotorisches Training mit dem Ziel der Optimierung der Rumpfstabilität
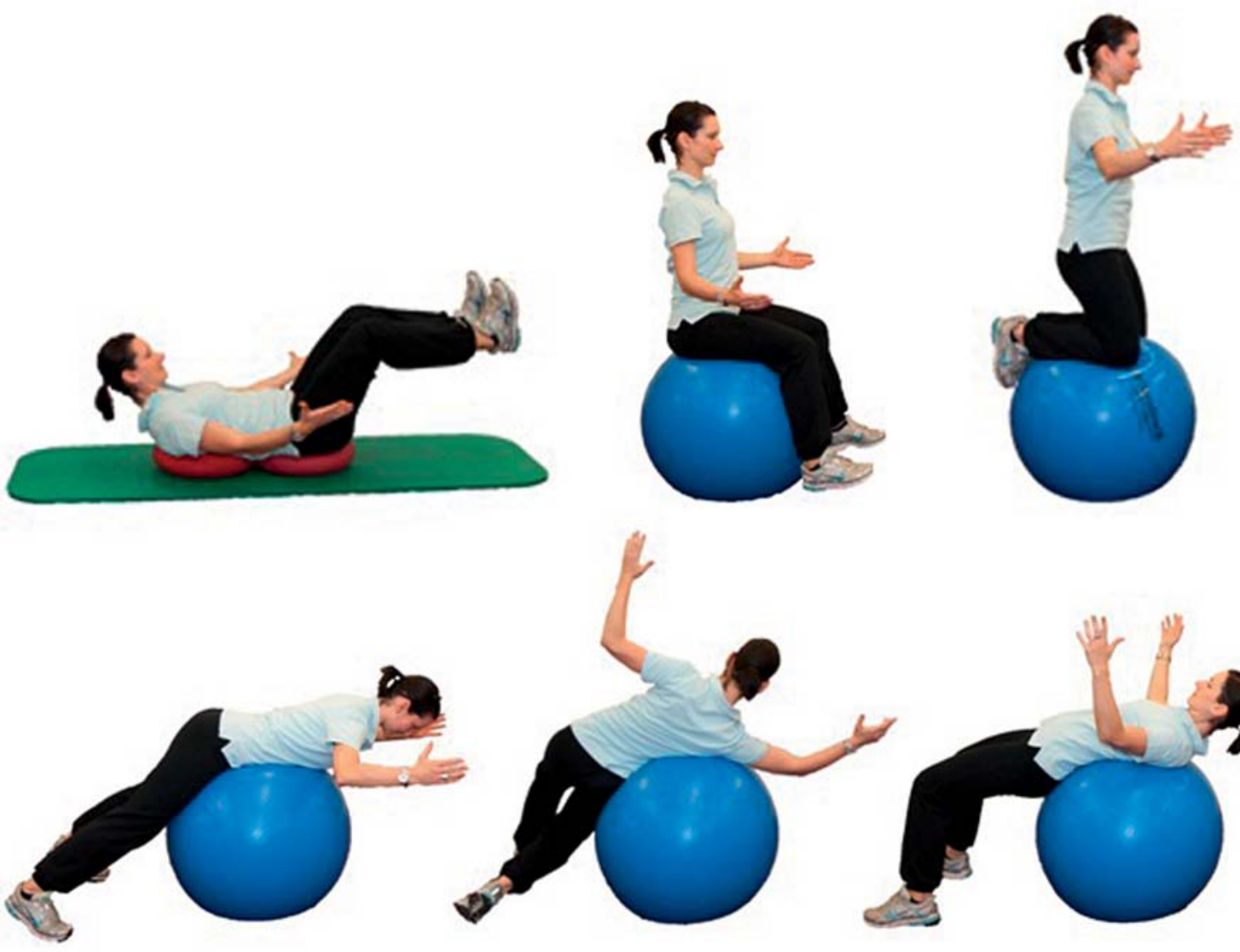

Elektrotherapie) sowie die neurophysiologisch orientierte Bewegungsschulung. In der weiteren physiotherapeutischen Behandlung von Sportlern kommen vorwiegend folgende Methoden bzw. Techniken zum Einsatz (Tab. 3):

- Manualtherapeutische Techniken,

- Massagetechniken (inkl. Querfriktionen und Lymphdrainage),

- Kryotherapie,

- Wärmetherapie (inkl. therapeutischem Ultraschall),

- Elektrotherapie.

Manualtherapeutische Techniken werden mit dem Ziel der Erkennung und Behandlung einer alterierten Muskel- und Gelenkfunktion angewandt. Bei Sportlern finden sich vorwiegend segmentale Hypomobilitäten der Wirbelsäule, der Rippengelenke und des Beckens. Nach suffizienter Behandlung ist die Gelenkfunktion häufig schnell wieder hergestellt. Der wissenschaftliche Beleg einer nachhaltigen Wirksamkeit im Längsschnitt bei
Athleten ist jedoch derzeit nicht abschließend geführt worden.

Zu den am häufigsten angewendeten passiven Methoden bei Athleten zählen unterschiedliche Massagetechniken. Vielfach werden Ganzkörpermassagen zu Regenerationszwecken der Muskulatur routinemäßig nach Trainingseinheiten durchgeführt, wenngleich ein regenerationsfördernder Effekt bislang nicht nachgewiesen werden konnte. Querfriktionen nach Cyriax haben in der Behandlung von Tendinopathien einen festen Stellenwert. Die regelmäßige Anwendung trägt nachweislich zur Erhöhung der Kollagennettosynthese bei und führt reflexinduziert zur Minderung der Schmerzen. Aufgrund der zum Teil sehr schmerzhaften Behandlung ist eine Vorbehandlung mit Eis, therapeutischem Ultraschall oder analgetischen Stromanwendungen empfehlenswert.

Im Therapieverlauf finden vermehrt sporttherapeutische (Medizinische Trainingstherapie, Sporttherapie) und später sportartspezifische Inhalte Berücksichtigung. 


\section{Hintergrund}

\section{Abb. 9 - Exzentrisches Training der kniegelenkstabilisierenden Muskulatur (1., 2.) und der Wadenmuskulatur/Achillessehne (3.)}

Der Extremitäten- und der Rumpfmuskulatur kommt neben der Notwendigkeit, das Körpergewicht zu überwinden (konzentrische Belastung) auch die Aufgabe zu, Bremsarbeit zu verrichten (exzentrische Belastung). Hierbei konnte nachgewiesen werden, dass neben einem Hypertrophiereiz für die Muskulatur höhere Kräfte bei geringerer Ermüdung und reduzierter metabolischer und kardiozirkulatorischer Belastung erreicht werden. Als ein Erklärungsmodell konnte ge- zeigt werden, dass additive, passive Elastizitätskräfte der serienelastischen Elemente des Muskel-SehnenApparats für die höhere Kraft verantwortlich sind. Aufgeklärt werden muss darüber, dass insbesondere ungewohnte exzentrische Belastungsformen anfänglich muskuläre, reversible Beschwerden - im Sinne eines Muskelkaters - nach sich ziehen können. Angewandt wird diese Form des Trainings entweder isoliert (z.B. für die Waden- oder Quadrizepsmuskulatur in der konservativen Behandlung von Patellasehnenoder Achillessehnentendinopathien oder in der Prävention von $\mathrm{ACL}$-Verletzungen für die Ischiokruralmuskulatur) oder kombiniert mit konzentrischen Belastungen als sog. Reaktivkrafttraining bzw. im Dehnungs-Verkürzungs-Zyklus (z. B. bei Sprüngen). Ein Trainingsumfang von mindestens 2 Einheiten pro Woche mit 3 $\times 8-10$ Wiederholungen pro Übung werden meist empfohlen.
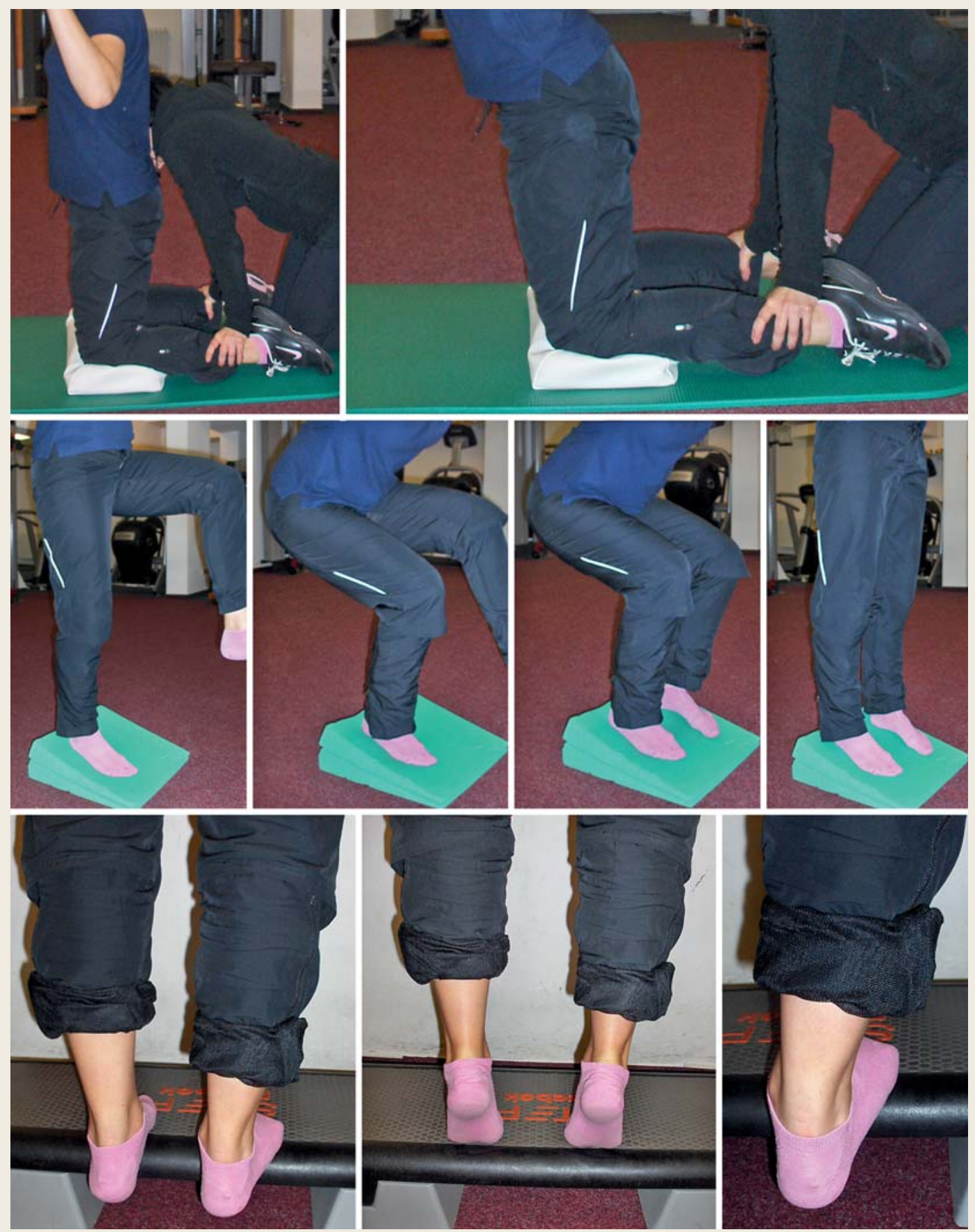

1. „Nordic Hamstrings“. Langsames Nach-vorneNeigen von Oberkörper und Oberschenkel bei fixiertem Unterschenkel.

2. Einbeinige Kniebeugen.

3. Beidbeiniger Stand, beidbeinige konzentrische Arbeit in den Zehenstand und einbeinige exzentrische Arbeit nach unten über die Horizontale hinaus. 
Mechanismen ausgewählter physikalischer Therapieformen (in Anlehnung an Hirschmüller, Mayer 2010).

\begin{tabular}{|c|c|c|c|}
\hline Applikation & Mögliche Effekte & Mögliche Wirkmechanismen & Eventuelle Nebenwirkungen \\
\hline $\begin{array}{l}\text { Massage } \\
\text { Streichung } \\
\text { Klopfung } \\
\text { Knetung } \\
\text { Friktion } \\
\text { Vibration }\end{array}$ & $\begin{array}{l}\text { Temperaturerhöhung (Haut und Muskel) } \\
\text { Senkung Muskeltonus } \\
\text { Schmerzreduktion } \\
\text { Entspannung } \\
\text { Abtransport von Stoffwechselprodukten }\end{array}$ & $\begin{array}{l}\text { Aktivierung der Zirkulation } \\
\text { (Haut und Muskel) } \\
\text { Lösen von Gewebeverklebungen } \\
\text { Erhöhung der Gleitfähigkeit der } \\
\text { Gewebeschichten } \\
\text { Erhöhung der Aktivität des } \\
\text { Parasympathikus }\end{array}$ & $\begin{array}{l}\text { Lokalreaktionen (z. B. Allergien auf Öle) } \\
\text { reaktive Schwellung, Hämatom } \\
\text { fehlende Schmerzhemmung }\end{array}$ \\
\hline $\begin{array}{l}\text { Wärmetherapie } \\
\text { Fango } \\
\text { Heatpacks } \\
\text { Whirlpool } \\
\text { Heißluft } \\
\text { Rotlicht }\end{array}$ & $\begin{array}{l}\text { Schmerzlinderung } \\
\text { Verbesserte Beweglichkeit } \\
\text { Abtransport von Stoffwechselprodukten } \\
\text { verbessert }\end{array}$ & $\begin{array}{l}\text { Stoffwechselsteigerung, Vasodilatation } \\
\text { Erhöhung der Dehnfähigkeit } \\
\text { (Bindegewebe, Kapseln) } \\
\text { Abnahme Muskel- und Sehnenspannung }\end{array}$ & $\begin{array}{l}\text { Verletzungsgefahr durch Anästhesie } \\
\text { Verbrennungen } \\
\text { Ödem } \\
\text { Schwellung } \\
\text { fehlende Schmerzhemmung }\end{array}$ \\
\hline $\begin{array}{l}\text { Kryotherapie } \\
\text { Coldpacks } \\
\text { Eiswasser } \\
\text { Eismassage }\end{array}$ & $\begin{array}{l}\text { Schmerzlinderung } \\
\text { Eindämmung von Schwellung und } \\
\text { Hämatombildung }\end{array}$ & $\begin{array}{l}\text { Hemmung der Nozizeptoren } \\
\text { Abnahme Nervenleitgeschwindigkeit } \\
\text { Minderung Zellstoffwechsel, } \\
\text { Vasokonstriktion }\end{array}$ & $\begin{array}{l}\text { Erfrierung } \\
\text { Nervenschädigung } \\
\text { Reaktive Hypervaskularisierung, Ödem }\end{array}$ \\
\hline $\begin{array}{l}\text { Ultraschall } \\
\text { kontinuierlich, } \\
\text { periodisch }\end{array}$ & $\begin{array}{l}\text { thermische Effekte } \\
\text { mechanische Effekte }\end{array}$ & $\begin{array}{l}\text { thermisch: s.,Wärme“ } \\
\text { mechanisch: Steigerung der } \\
\text { Zellpermeabilität } \\
\text { Anregung des Zellmetabolismus } \\
\text { Schmerzlinderung }\end{array}$ & $\begin{array}{l}\text { Verbrennung } \\
\text { Lokalreaktionen (Allergie, Hautreizung) } \\
\text { Schwellung } \\
\text { fehlende Schmerzhemmung }\end{array}$ \\
\hline $\begin{array}{l}\text { Elektrotherapie } \\
\text { Hochfrequenzstrom }(>100 \mathrm{kHz}) \\
\text { Mittelfrequenzstrom }(1-100 \mathrm{kHz}) \\
\text { Niederfrequenzstrom }(1-1000 \mathrm{~Hz})\end{array}$ & $\begin{array}{l}\text { Schmerzlinderung } \\
\text { Ödemeindämmung } \\
\text { Entzündungshemmung } \\
\text { Muskelstimulation }\end{array}$ & $\begin{array}{l}\text { Erwärmung } \\
\text { Blockierung schmerzleitender } \\
\text { Nervenfasern } \\
\text { Detonisierung }\end{array}$ & $\begin{array}{l}\text { Verbrennung } \\
\text { Lokalreaktionen (Allergie, Hautreizung) } \\
\text { Schwellung } \\
\text { fehlende Schmerzhemmung }\end{array}$ \\
\hline
\end{tabular}

Die Medizinische Trainingstherapie (MTT) ist dabei als inhaltliche Schnittstelle zwischen Physiotherapie und Sporttherapie zu sehen. Hauptziele in MTT und Sporttherapie sind - neben der Zunahme des Muskelquerschnitts - die Optimierung der Flexibilität, die Verbesserung der Muskelfunktion und die Förderung der neuromuskulären Kontrolle (u. a. durch exzentrische Belastungsformen, s. Hintergrundbox). Ein neuromuskulär fokussiertes Training zeigte hierbei sowohl auf dem Gebiet des Krafttrainings als auch des sensomotorischen Trainings bereits früh positive Effekte (auch bei Hochleistungsathleten). So konnte beispielsweise im Rahmen einer randomisierten, kontrollierten Untersuchung (522 Athleten, maximal 2 Monate zurückliegendes Supinationstrauma) nachgewiesen werden, dass ein 8-wöchiges sensomotorisches Training, selbstständig im Längsschnitt über 1 Jahr durchgeführt, eine Risikoreduktion von $35 \%$ für das erneute Auftreten eines Supinationstraumas nach sich zieht (Hupperets et al. 2009). Ähnliche Daten sind für eine Reduktion des Wiederauftretens von Rückenschmerzen nach einem Krafttraining bekannt (Hayden et al. 2005). Im letzten Schritt des Therapieprozesses werden sportartspezifische Handlungsmuster simuliert, die darauf abzielen, den Athleten an die in der jeweiligen Sportart vorhandenen Belastungen (erneut) zu adaptieren. Der Erfolg konservativer Therapiekonzepte in Physio- und Sporttherapie ist maßgeblich an eine regelmäßige Durchführung gekoppelt. Die Häufigkeit der Anwendungen in der Rehabilitation von Sportverletzungen sollte - abhängig von Pathologie, Behandlungsstadium und Überlastungsbeschwerden -2 bzw. 3 Behandlungen pro Woche nicht unterschreiten.

- Hauptziele in MTT und Sporttherapie sind die Verbesserung der Muskelfunktion und die Förderung der funktionellen Gelenkstabilität unter Belastung.

\section{Einlagen und Schuhe}

Der Einsatz von Schuheinlagen im Sport ist zur Behandlung belastungsbedingter Beschwerden der unteren Extremität weit verbreitet. Einige hochwertige Studien belegen die Wirkung von individuell angepassten Sport- 
einlagen auf Überlastungsbeschwerden der unteren Extremität. Besonders wirksam zeigte sich der Einsatz von Sporteinlagen in der Therapie von Tendinopathien der Achilles- und Patellasehne, der Plantarfasziitis, dem femoropatellaren Schmerzsyndrom und dem Tibiakantensyndrom sowie dem Tractus-iliotibialis-Syndrom.

Als Basis für die Herstellung von Sporteinlagen hat sich in den vergangenen Jahren die dynamische Ganganalyse (Pedografie) mit Messung der plantaren Druckverteilung durchgesetzt. Im Gegensatz zum häufig verwendeten statischen Fußabdruck im Trittschaum kann so die Dynamik des Abrollvorgangs mit muskulärem Ausgleich statischer Fußfehlformen beurteilt werden. Die Anpassung der Einlage erfolgt damit an die funktionelle Geometrie des Fußes während der Abrollbewegung mit dreidimensionaler Ausformung von wirksamen Funktionselementen. Die eingesetzten Materialen zeichnen sich durch eine höhere Flexibilität bei ausreichender Stabilität aus (z. B. Polyurethan). Die horizontale Bauweise (Sandwich-Bauweise) wird zunehmend durch vertikale Fertigungstechniken von Rohlingen, die nachfolgend individuell zugeschliffen werden können, ersetzt. Dies führt zur Verbesserung von Stabilität und Haltbarkeit der Einlage. Unterschiedliche Funktionselemente wie Weichbettungen, Keile oder Gewölbestützen werden bereits in die ursprüngliche Roheinlage eingearbeitet. Weichbettungen werden effektiv bei akuten Beschwerden (z. B. Metatarsalgie, Hallux valgus oder Plantarfasziitis) eingesetzt. Über eine erhöhte Schalenform im Rückfußbereich wird eine verbesserte Fußführung erreicht. Im Vorfußbereich eingearbeitete Detorsions- bzw. Supinationskeile zielen auf eine Modifizierung beim finalen Fußabdruck ab.

Die zugrunde liegenden Wirkmechanismen von Sporteinlagen sind bislang nur unzureichend geklärt. Der Effekt scheint vorwiegend auf einer Modulation des afferenten Inputs oder Anpassungen der Muskelaktivität zu basieren. Aktuelle wissenschaftliche Untersuchungen mit elektromyografischen Messungen bestätigen den Einfluss dieser sensomotorischen Regulationsmechanismen. So wurde unter therapeutischer Verwendung einer Längsgewölbestütze eine nachhaltige Änderung der Muskelaktivität des wichtigsten Sprunggelenksstabilisators (M. peronaeus longus) erreicht (Baur et al. 2011).

Uneinheitlich wird ein möglicher Einsatz (bzw. Wechsel) von Sportschuhen in der Therapie von Überlastungsbeschwerden diskutiert. Bei Läufern wurde bis vor wenigen Jahren eine verstärkte Pronation des Fußes mit nachfolgender vermehrter Innenrotation des Unterschenkels als eine der Hauptursachen in der Entstehung von Überlastungsbeschwerden diskutiert. Aus diesem Grund wurde die Nutzung eines Laufschuhs mit Pronationsstütze häufig propagiert. Eine Reduktion der Beschwerden durch Einsatz einer Pronationsstütze konnte jedoch nicht nachgewiesen werden. Somit werden Stützen zur Einschränkung von Pro- oder Supination lediglich im Einzelfall und individuell bei symptomatischen Patienten mit pathologischem Abrollmuster (z. B. beim symptomatischen, muskulär nicht kompensierbaren Knick-Spreiz-Fuß) empfohlen. Asymptomatische Läufer sollten hingegen bevorzugt Neutralschuhe, ohne zusätzliche Stützen, tragen.

Sehr weich gedämpfte Schuhe können durch Erhöhung des Stabilisationsbedarfs akute Belastungsreaktionen auslösen, was sich häufig in Form von Achillesoder Patellasehnenbeschwerden äußern kann. Zu hart gedämpfte Sportschuhe bewirken dagegen eine Fortleitung der Kräfte bevorzugt auf die vordere Loge der Unterschenkelmuskulatur, was klinisch beispielsweise in Form eines Tibiakantensyndroms beschrieben ist. In Mannschafts- und Rückschlagsportarten sind die Sportschuhe aufgrund der lateral-dynamischen Bewegungsmuster vorwiegend durch dünne Zwischensohlen geprägt. Dies führt zu geringeren Scherkräften auf das Sprunggelenk und bietet eine höhere Flexibilität für schnelle, kleinschrittige Bewegungen mit häufigen Richtungswechseln. Wissenschaftlich validierte Konzepte für die Therapie von Sportverletzungen und Überlastungsbeschwerden sind hier allerdings derzeit nicht verfügbar.

- Individuell angefertigte Sporteinlagen können in der Behandlung muskuloskelettaler Überlastungsbeschwerden sinnvoll sein. Bezüglich der Sportschuhversorgung stehen wissenschaftlich validierte Konzepte noch aus.

\section{Validierte Konzepte zur Prävention von Verletzungen und Überlastungs- beschwerden im Sport}

Die Prävention von Sportverletzungen und Überlastungsbeschwerden wird über unterschiedliche Konzepte verfolgt, wobei nur einige Strategien abschließend evidenzbasiert belegt sind (Steffen et al. 2010; Tab. 4). Die Mehrzahl der Untersuchungen in den letzten Jahren befasste sich mit der Prävention von ACL-Verletzungen (insbesondere bei Athletinnen), der Prävention von Supinationstraumata als häufigster Sportverletzung und der Prävention von Rückenschmerzen (u.a. über den Einsatz körperlicher Aktivität als Intervention). Sinnvollerweise erfolgt eine Beurteilung präventiver Möglichkeiten geordnet nach der Lokalisation. 


\section{Grundlagen}

Tabelle 4

Beispiel eines Trainingsprogramms zur Prävention von Verletzungen der oberen Extremität im Sport (Übungsauswahl, Ausführung und Dosierung).

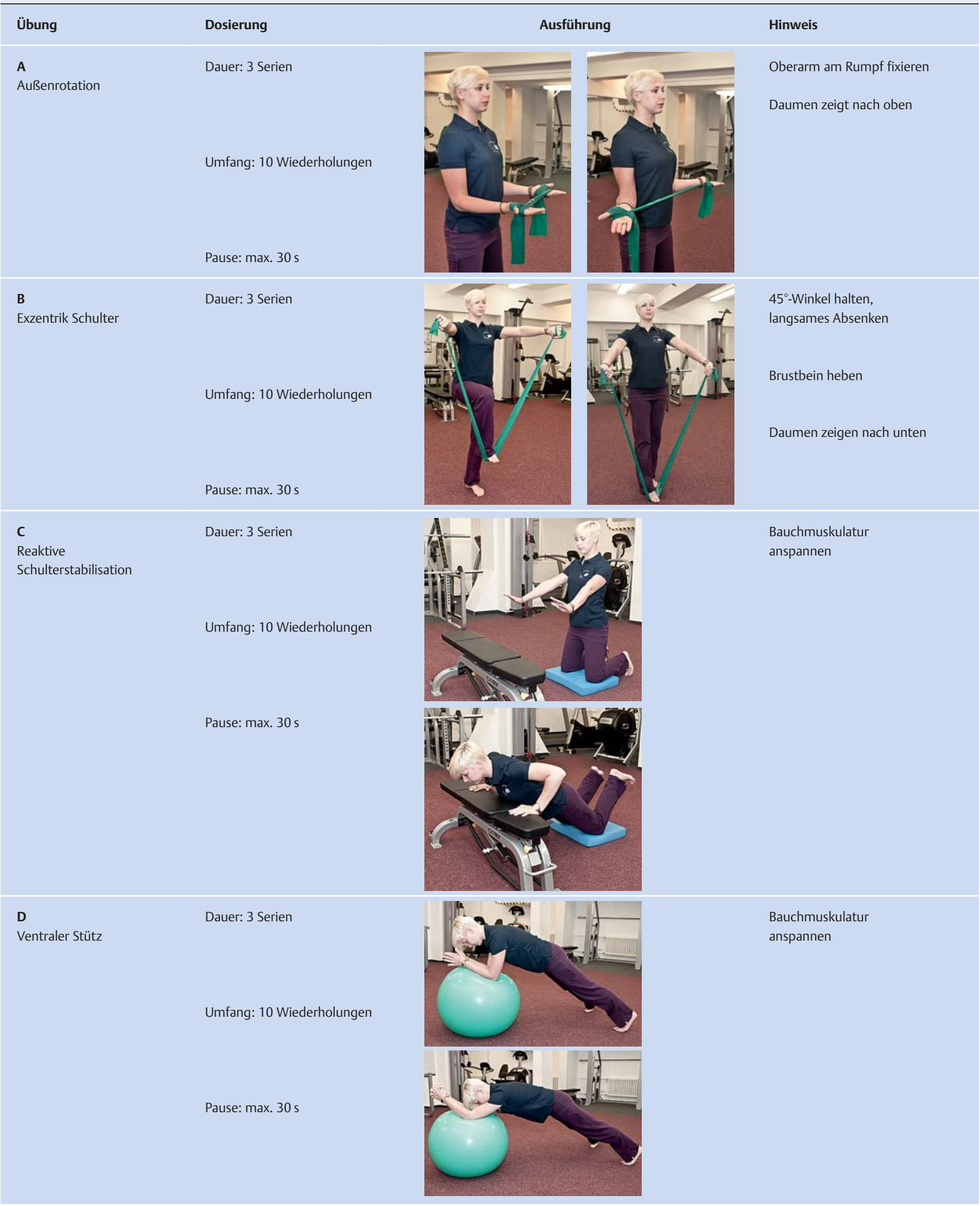




\section{Kopf, Hals und obere Extremität}

Die Mehrzahl der Kopfverletzungen im Sport ist von geringer Intensität und Dauer und tritt selten auf. Dennoch werden in Einzelfällen (insbesondere bei Stürzen und Kontaktunfällen) schwere Schädel-Hirn-Traumata beobachtet. Häufig sind Gehirnerschütterungen (z. B. durch Kopfballsituation im Fußball), denen v.a. bei Kindern und Jugendlichen ein erhöhtes Augenmerk geschenkt werden sollte. Eine Risikoreduktion von bis zu $80 \%$ ist durch das Tragen von Helmen in Sportarten mit hohem Risiko für Kopfverletzungen wie alpiner Skilauf und Radsport belegt.

Verletzungen des Schultergürtels im Sport treten meist bei hoher Geschwindigkeit und/oder einer Sturzsituation auf. Eine Reduktion von Gegnerkontakt (z.B. über eine Regelanpassung in Spielsportarten), eine Vermeidung von Stürzen und die Aufrechterhaltung eines suffizienten Muskelkorsetts gelten somit als wichtige Präventionsstrategien. Für die Schulterluxation kann derzeit keine gesicherte Intervention zur Risikoreduktion angegeben werden. Ähnlich ist die Datenlage bezüglich Ellenbogenverletzungen und Überlastungsbeschwerden. Bezogen auf Finger, Hand und Handgelenke haben sich Protektoren für das Auftreten von (Wieder-)Verletzungen bewährt. Eine Reduktion der Verletzungsrate von rund $10 \%$ ist bekannt. Tape-Verbände der Finger können insbesondere das Wiederauftreten von Kapsel-Band-Verletzungen reduzieren.

\section{Wirbelsäule und Becken}

Bezüglich Rückenbeschwerden wird in verschiedenen Metaanalysen und internationalen Leitlinien postuliert, dass körperliche Aktivität zur Prävention vorteilhaft ist (hoher Evidenzgrad bei moderater Effektstärke), jedoch kann keine Überlegenheit einer Methode angegeben werden. Eine additive Kombination mit verhaltenstherapeutischen Techniken, insbesondere für chronifizierte Rückenbeschwerden, ist darüber hinaus evident (Choi et al. 2010). Mit Blick auf die akute, mittel- und langfristige Belastbarkeit, sowohl von Spitzenathleten als auch der Belastbarkeit im Alltag, kommt der Stabilität der Wirbelsäule und der Adaptationsfähigkeit der Muskulatur in differenzierten Belastungssituationen eine vorrangige Bedeutung zu. Dies betrifft die Optimierung der Kraftleistungsfähigkeit und der sensomotorischen Funktionsfähigkeit der Rumpfmuskulatur. Als besonders bedeutsam wird hierbei die neuromuskuläre Kontrolle der Wirbelsäule unter Last angesehen. Darunter wird die Fähigkeit verstanden, das dynamische Gleichgewicht der einzelnen Segmente trotz wechselnder mechanischer Störungen und/oder neuromuskulärer Kontrollfehler aufrecht zu erhalten.
- Körperliche Aktivität ist sowohl in der Therapie als auch der Prävention chronisch-unspezifischer Rückenschmerzen validiert. Zudem wird die Dauer bis zum Wiedereintritt der Beschwerden erhöht und deren Häufigkeit reduziert.

Nicht selten treten Muskel- und Ansatzverletzungen/ -beschwerden am Becken auf (rund 20\% aller Muskelverletzungen). Prädestiniert sind Sportarten mit hoher Bewegungsgeschwindigkeit und plötzlicher Belastung (z. B. Eishockey, Fußball, Leichtathletik). Belegt ist, dass eine konsequente Ausheilung aller (Vor-)Beschwerden und (Vor-)Verletzungen der Becken- (und Hüft-)region eine obligate Präventionsstrategie für erneute Verletzungen darstellt. Darüber hinaus wird die Optimierung der (exzentrischen) Kraftleistungsfähigkeit mit einer folgenden Verbesserung der funktionellen Beckenstabilität als sinnvoll erachtet.

\section{Untere Extremität}

Bei Sportverletzungen der unteren Extremität stehen Läsionen des Bandapparats des oberen Sprung- und des Kniegelenks sowie Verletzungen der Oberschenkelmuskulatur im Vordergrund. Es konnte nachgewiesen werden, dass ein multimodales Training (u. a. Kraft und Sensomotorik) unter der Voraussetzung einer hohen Compliance zu einer Risikoreduktion, insbesondere für akute Knie- und Sprunggelenk(wieder)verletzungen, führt (Hübscher et al. 2010).

Die Prävention von Knieverletzungen fokussiert seit einigen Jahren auf eine Risikoreduktion von vorderen Kreuzbandverletzungen. Etwa 70\% der ACL-Rupturen treten dabei ohne Gegnerkontakt auf. Bedeutsam sind Stoppbewegungen, Richtungswechsel und Landungen bei stehendem Fuß mit nahezu gestrecktem Kniegelenk sowie Stürze nach hinten bei gebeugtem Kniegelenk. Somit werden eine adäquate Technik bei Landungen und Belastungen unter Richtungswechsel, eine suffiziente sensomotorische Leistungsfähigkeit der das Kniegelenk umgreifenden Muskulatur und eine hohe exzentrische Kraftleistungsfähigkeit (ischiokrurale Muskulatur, Streckapparat, Hüftabduktoren) als Präventionsziele verfolgt.

Eine norwegische Arbeitsgruppe überprüfte in diesem Zusammenhang ein Trainingsprogramm, bestehend aus 11 Übungen, zur Optimierung der Rumpfstabilität und Balancefähigkeit, der dynamischen Stabilisationsfähigkeit der Gelenke und der exzentrischen Kraftleistungsfähigkeit der Ischiokruralmuskulatur an Fußballerinnen. Als Ergebnis fand sich eine geringere Verletzungsanzahl und eine geringere Anzahl an Überlastungsbeschwerden in der Interventionsgruppe gegenüber der Kontrollgruppe (Soligard et al. 2008). Beidbeinige Landungen werden empfohlen, wo immer sie 
sportartspezifisch möglich sind, eine exzessive, dynamische Valgusposition ist zu vermeiden. Vergleichbare Ergebnisse zeigte das sogenannte PEP-Programm ( $\mathrm{PEP}=$ Prevent Injuries and Enhance Performance) einer amerikanischen Arbeitsgruppe (Gilchrist et al. 2008). Das Aufwärmprogramm beinhaltet Kräftigungsübungen, Stretching, sportartspezifische Laufwege, Richtungswechsel und plyometrische Trainingsanteile. Die Verletzungsrate in der Interventionsgruppe war 1,7-fach niedriger verglichen mit der Kontrollgruppe. Neuere Ansätze weisen zudem darauf hin, dass eine erhöhte Rumpfstabilität eine Risikoreduktion von Knieverletzungen nach sich zieht.

Die häufigste akute Verletzung unter sportlicher Belastung betrifft den fibulotalaren Kapsel-Band-Apparat (rund 25\% aller Sportverletzungen), wobei eine zurückliegende Vorverletzung und eine nicht ausreichende sensomotorische Stabilisationsfähigkeit als Risikofaktoren gelten. In rund 30\% der Fälle resultiert eine chronische Sprunggelenkinstabilität. Aus präventiver Sicht ist somit ein sensomotorisches Training (z.B. auf unebenen oder weichen Untergründen, Sprünge), auch unter zusätzlicher Last, sinnvoll und durch verschiedene Untersuchungen belegt. Mehrere Arbeiten weisen auf den Nutzen und den sinnvollen Einsatz externer Stabilisierungshilfen hin, wenngleich eine Risikoreduktion durch Sprunggelenkbandagen oder Tape-Verbände nur bei Athleten mit einer Vorverletzung abschließend belegt ist.

\section{Literatur}

Alonso JM, Junge A, Renström P et al. Sports injuries surveillance during the 2007 IAAF World Athletics Championships. Clin J Sport Med 2009; 1: 26-32

Baur H, Hirschmüller A, Müller S et al. Neuromuscular activity of the peroneal muscle after foot orthoses therapy in runners. Med Sci Sports Exerc 2011; 43: 1500 - 1506

Bono CM. Low-back pain in athletes. JBJS-Am 2004; 86-A: 382 - 396

Choi BK, Verbeek JH, Tam WW et al. Exercises for prevention of recurrences of low-back pain. Cochrane Database Syst Rev 2010, 1. Art. No. CD006555. DOI: 10.1002/14651858.CD006555.pub2

Gilchrist J, Mandelbaum BR, Melancon $\mathrm{H}$ et al. A randomized controlled trial to prevent anterior cruciate ligament injury in female collegiate soccer players. Am J Sports Med 2008; 36: 1476- 1483

Hayden JA, van Tulder MV, Malmivaara A et al. Exercise therapy for treatment of non-specific low back pain. Cochrane Database Syst Rev 2005, 3. Art. No. CD000335. DOI: 10.1002/14651858.CD000335. pub2

Hirschmüller A, Mayer F. Grundlagen und Anwendungen physikalischer Therapiemaßnahmen im Sport. In: Dickhuth HH, Mayer F, Röcker K et al. (Hrsg). Sportmedizin für Ärzte. 2. überarbeitete Auflage. Köln: Deutscher Ärzteverlag, 2010

Hübscher M, Zech A, Pfeifer K et al. Neuromuscular training for sports injury prevention: a systematic review. Med Sci Sports Exerc 2010; 42: $413-421$

Hupperets MD, Verhagen EA, van Mechelen W. Effect of unsupervised home based proprioceptive training on recurrences of ankle sprain: randomised controlled trial. BMJ 2009; 339: b2684 doi: 10.1136/ bmj.b2684

Junge A, Engebretsen L, Mountjoy $\mathrm{M}$ et al. Sports injuries during the Summer Olympic Games 2008. Am J Sports Med 2009; 37: 2165 2172

McKeon PO, Mattacola CG. Interventions for the prevention of first time and recurrent ankle sprains. Clin Sports Med 2008; 27: 371 - 382

Renström P, Ljungquist A, Arendt $E$ et al. Non-contact $A C L$ injuries in female Athletes: an International Olympic Committee current concepts statement. Br J Sports Med 2008; 42: 394-412

Soligard T, Myklebust G, Steffen K et al. Comprehensive warm-up programme to prevent injuries in young female footballers: cluster randomized controlled trial. BMJ 2008; 337: a2469 doi: 10.1136/bmj. a2469

Steffen K, Andersen TE, Krosshaug T et al. ECSS Position Statement 2009: Prevention of acute sports injuries. Eur J Sport Sci 2010; 10: 223 -236

\section{Korrespondenzadresse}

Prof. Dr. med. Frank Mayer

Hochschulambulanz der Universität Potsdam

Professur für Sportmedizin und Sportorthopädie

Am Neuen Palais 10, Haus 12

14469 Potsdam

Telefon: 0331/977-1768

Telefax: 0331/977-1296

E-Mail: fmayer@uni-potsdam.de 


\section{CME-Fragen}

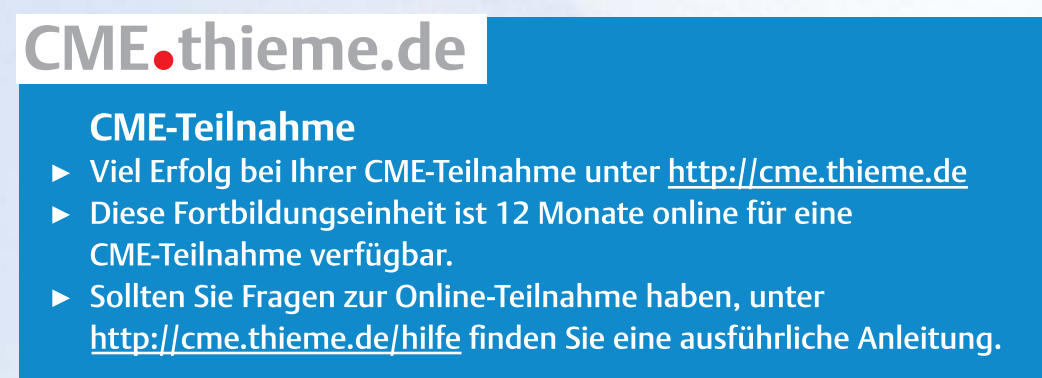

Als häufigste Verletzung
im Sport gilt...

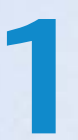

A die Rotatorenmanschettenruptur.

B die Kapsel-Band-Verletzung des oberen Sprunggelenks.

C die Radiusfraktur.

D die vordere Kreuzbandruptur.

E die Patellaluxation.

Welche Aussage ist richtig?

Im Nachwuchs-

leistungssport...
A sind ACL-Rupturen v. a. bei männlichen Athleten evident.

B ist die Rate von Überlastungsschäden höher als bei Erwachsenen.

C ist sportbedingten Gehirnerschütterungen besondere Beachtung zu schenken (längere Regeneration).

D sind v.a. Rückenschmerzen häufig.

E treten Apophysen- und Epiphysenverletzungen besonders selten auf.
Welche Aussage ist richtig?

Chronisch unspezifische Rückenschmerzen ...
A lassen sich mittels MRT eindeutig nachweisen.

B sind im Spitzensport wesentlich häufiger als in der Allgemeinbevölkerung.

C gehen auf eine Degeneration der Bandscheiben zurück.

D sind meist Folge einer akuten Sportverletzung.

E treten im Spitzensport mit einer jährlichen Erstinzidenz von rund 5\% auf.
Welche Aussage ist richtig?

Tauglichkeits- und Jahresgrunduntersuchungen von Spitzenathleten mit Kaderstatus ...
A dienen der Feststellung der Leistungsfähigkeit der Athleten.

B werden meist vom Hausarzt durchgeführt.

C beinhalten immer ein MRT der unteren Wirbelsäule.

D werden üblicherweise an lizensierten Untersuchungszentren des DOSB durchgeführt.

E finden nur bei Beschwerden der Athleten statt.
Welche der folgenden Methoden sind in der Untersuchung von Athleten obligat?
A medizinische Anamnese, Trainingsanamnese und körperliche Untersuchung

B Kraftmessungen der oberen Extremität

C Röntgenaufnahmen der LWS in 2 Ebenen

D Erfassung der posturalen Kontrolle

E Sonografie der Achilles- und Patellasehne 


\section{Grundlagen}

Welche der folgenden
Therapieformen werden
bei Sportlern selten

Welche Aussage ist richtig?

Unter einem sensomotorischen Training versteht man ...

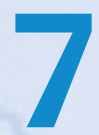

A die Zunahme der Muskelmasse durch Krafttraining.

B die Einflussnahme auf afferente Information mit dem Ziel einer definierten motorischen Antwort.

C die Optimierung der intramuskulären Koordination.

D die Erhöhung des Bewegungsausmaßes eines Gelenks.

E eine Trainingsmaßnahme zur kurzfristigen Schmerzreduktion.
Welche der folgenden

Präventionsmaß-

nahmen sind nicht

sinnvoll?
A Tragen eines Helms im alpinen Skisport

B Krafttraining zur Erhöhung der funktionellen Gelenkstabilität

C körperliche Aktivität zur Vermeidung von Rückenschmerzen

D regelmäßige Schonung zur Reduktion von Überlastungsschäden

E Ausheilen einer Verletzung zur Risikoreduktion einer Wiederverletzung
Welche Faktoren sind für eine Erhöhung des Verletzungsrisikos im Sport nicht validiert?
A Bergläufe und Tempoeinheiten

B eine Erhöhung des Laufumfangs über $60 \mathrm{~km} /$ Woche für Laufsportbeschwerden

C mehrere Vorverletzungen

D nicht ausgeheilte Verletzungen

E inadäquate Landetechnik für ACL-Rupturen bei weiblichen Nachwuchsathletinnen
Welche Aussage trifft nicht zu?

Exzentrische Belastungen ...

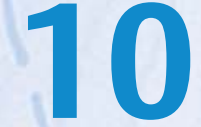

A sind in der Sporttherapie sinnvoll einzusetzen.

B lösen eine hohe kardiovaskuläre und metabolische Reaktion aus.

C können Muskelkater erzeugen.

D erzeugen hohe Kräfte.

E sind Teil des Dehnungs-Verkürzungs-Zyklus. 